\title{
Practical Earthquake Location on a Continental Scale in Australia Using the AuSREM 3D Velocity Model
}

\author{
by M. de Kool and B. L. N. Kennett
}

\begin{abstract}
This study explores the full 3D earthquake location for the Australian continent, exploiting the recent 3D Australian Seismological Reference Model (AuSREM). Seismic velocities from AuSREM were used as input to precompute finely spaced $P$ - and $S$-travel-time grids for each station in the Australian National Seismograph Network using the multistage fast marching method. Travel times from anywhere in the grid to the corresponding station can then be computed by interpolation. The location search using these travel times is based on matching observed and computed arrival times using the neighborhood algorithm. All computations involved can be performed in practical time frames on a single processor computer.

The performance of the 3D approach relative to location using the 1D global ak135 velocity model was assessed by locating a set of recent earthquakes. The arrival-time residuals for $P$ and $S$ arrivals are significantly smaller when using the 3D AuSREM model. The improvements over ak135 are especially large in the $10^{\circ}-18^{\circ}$ distance range, in which a distance bias is strongly reduced and for those paths where the ak135 residuals are large. A small set of six ground-truth events was used to assess to what extent the reduction in travel-time residuals leads to better absolute location accuracy. The 3D location offset from the ground-truth position is typically half that of the ak135 offset. The patterns of offsets suggest that the already fast mantle wavespeeds in western Australia need to be even faster than in AuSREM.
\end{abstract}

\section{Introduction}

Earthquake location using standard global 1D reference models such as IASP91 (Kennett and Engdahl, 1991) and ak135 (Kennett et al., 1995) can achieve good results in both teleseismic and regional applications. Nevertheless, it has long been recognized that the level of heterogeneity in the lithosphere is too large to be ignored. In cratonic regions with fast wavespeeds in the mantle lithosphere, $P$ arrivals beyond $10^{\circ}$ can arrive more than $5 \mathrm{~s}$ earlier than predicted by global models. Errors in location for well-characterized events using the global models will frequently exceed $10 \mathrm{~km}$, and the true location is likely to lie outside the formal error ellipse. A common approach has been the development of local 1D models. This is an effective approach when all stations used for location lie in the same geologic province as the earthquake, which typically means no more than a few hundred kilometers from the source. However, where strong contrasts in lithospheric properties are present across the region spanned by stations, a single 1D model is no more satisfactory than using a global reference model. Differences in seismic structure extend through the transition zone (Kennett, 2006) and can have a major influence on travel times in the epicentral distance range from $15^{\circ}$ to $25^{\circ}$.
Improved accuracy in earthquake location can be achieved by working directly in 3D, but this requires a substantial investment of effort: a 3D velocity model has to be constructed by inversion of information from many sources, and an efficient way to compute seismic travel times through such 3D models has to be available to perform routine earthquake location procedures. As a result, 3D location was initially applied in a research context for a small patch (e.g., volcanoes, Lomax et al., 2001) and areas at most a few hundred kilometers in size (e.g., Husen et al., 2003; Chen et al., 2006). At least one operational system for regional earthquake warning that can use 3D velocity models has just become available (Satriano et al., 2011). For earthquake location on a larger scale, major efforts have recently been undertaken in the nuclear monitoring community to construct global velocity models to be used for improved traveltime computation and hence location accuracy (e.g., SALSA3D, Begnaud et al., 2011; LLNL-G3D, Simmons et al., 2012). Such velocity models are derived by inverting observed travel-time residuals for a very large set of carefully relocated seismic sources, building on results using global reference models and regional corrections. In the long term, 
this style of work is clearly desirable. However, very large 3D models are not easy to implement because of the size and complexity of the models and the computational expense for travel-time calculations. Furthermore, these global 3D models can lack resolution and/or quality in some areas that are not well sampled by the ray paths of the seismic sources used in the inversion for the model. Additional information from alternative styles of analysis (e.g., surface-wave dispersion, active seismic experiments) can be used to infill and improve the quality of a 3D model. Such models need to encompass both the crust and the mantle components of the lithosphere (e.g., Molinari and Morelli, 2011; Schivardi and Morelli, 2011; Kennett and Salmon, 2012).

For event location at a continental scale, a broad range of epicentral distances need to be considered, particularly the range $15^{\circ}-23^{\circ}$ where the influence of lithospheric structure gives rise to major deviations from the predictions of the global 1D models (e.g., Kennett, 2006; Begnaud et al., 2011). For this reason, using a 3D model that provides information at these distances can be expected to give a greater improvement in location than a local 3D model that only provides travel times for phases traveling through the crust and uppermost mantle.

The continental lithosphere has been assembled through complex tectonic processes that can juxtapose material of very different ages and composition. Three-dimensional heterogeneity is most obvious in regions with active collisional processes in modern orogens, such as in the Himalaya in Asia, but is retained from older events. A striking example is the strong contrast in the mantle lithosphere across the Tornquist-Teisseyre line in Europe (see e.g., Schivardi and Morelli, 2011). North America shows very strong contrasts between the ancient continental core and the tectonically active zone to the west of the Cordillera (see e.g., Yuan and Romanowicz, 2010). Although Australia has seen little tectonic activity since $300 \mathrm{Ma}$, it retains a complex pattern of lithospheric elements with strong variations in seismic wavespeeds (e.g., Kennett and Salmon, 2012). Methods developed for improving earthquake location in Australia can therefore be of broad utility in other continental environments.

Figure 1 illustrates the way in which variations in structure across the Australian continent influence the passage times of seismic waves in the lithosphere. The paths between source and station are color coded by their residuals from the ak135 model for both $P$ and $S$ waves. The paths are plotted for events with magnitude $>5.5$ that can be relatively accurately located using teleseismic phases. For both $P$ and $S$ phases, most of the western and northern central parts of Australia show early arrivals. Compared with the ak135 model, the deviations are considerable with arrivals up to $20 \mathrm{~s}$ early for $S$. The size of such residuals is so large that even good phase readings are commonly dropped in standard analysis.

In the uppermost mantle in southern central Australia, there is a zone of slower arrivals in both $P$ and $S$ for energy traveling just below the thick crust in this region. As the epicentral distance increases, $P$ paths to the west and south from the same sources become very fast with rather early arrivals. With such large, and laterally variable, deviations from ak135, it is clearly desirable to build in the contrasts in the lithosphere beneath Australia into the earthquake location procedure so that observations with a broad span of epicentral distances can be effectively exploited. In this work, 3D location is applied at the continental scale using the 3D Australian Seismological Reference Model (AuSREM) for the structure of the Australian region down to $300 \mathrm{~km}$ (Kennett and Salmon, 2012).

The purpose of the paper is twofold. It investigates the practicality and benefits of seismic location procedures based on the 3D AuSREM velocity model for events on the Australian continent, and at the same time it provides an independent validation of the AuSREM model itself. In the following sections, the AuSREM velocity model and the computational methods are discussed briefly because the details have already been published elsewhere. The fully nonlinear 3D location procedure is then applied to a set of Australian earthquakes with good geographical coverage of the continent and additionally to a small set of ground-truth (GT) events.

\section{The 3D Velocity Model AuSREM}

The 3D velocity model used in this paper is the AuSREM. Kennett and Salmon (2012) provide an overview of the model and its construction. The crustal component of the model is presented by Salmon, Kennett, and Saygin (2013), and the details for the mantle component are given in Kennett et al. (2013). AuSREM is a 3D model defining $P$ and $S$-wave velocities and density on two grids, one for the crust and the other for the mantle down to a depth of $300 \mathrm{~km}$. In addition, a 2D grid of depth to Moho (Kennett et al., 2011; Salmon, Kennett, et al., 2013) is provided to separate the crust and mantle. The primary information for the crust is $P$ wavespeed, and all other parameters are derived from these values (Salmon, Kennett, and Saygin, 2013). For the mantle the primary information is the $S V$ wavespeed (Kennett et al., 2013). The various parameter values are specified at the grid nodes, and values at any point can be extracted by interpolation. The horizontal spacing of the grid nodes is $0.5^{\circ}$ in latitude and longitude for both crust and mantle. The basic crustal grid has $5 \mathrm{~km}$ vertical spacing, the mantle grid $25 \mathrm{~km}$ vertical spacing.

The AuSREM crustal model has been derived by combining data from many different sources, such as receiver functions, crustal tomography, refraction, and reflection surveys. The primary constraint on the mantle model comes from surface-wave tomography with some control also from regional body-wave tomography. $P$ - and $S$-travel-time observations for Australian earthquakes have not been used to constrain the model, so that the results of our present study can be seen as a truly independent test of its validity.

A minor modification of the AuSREM model was required to account for an inconsistency between the Moho depth and velocity models, which arises because these two data sets had been derived independently. In particular, 

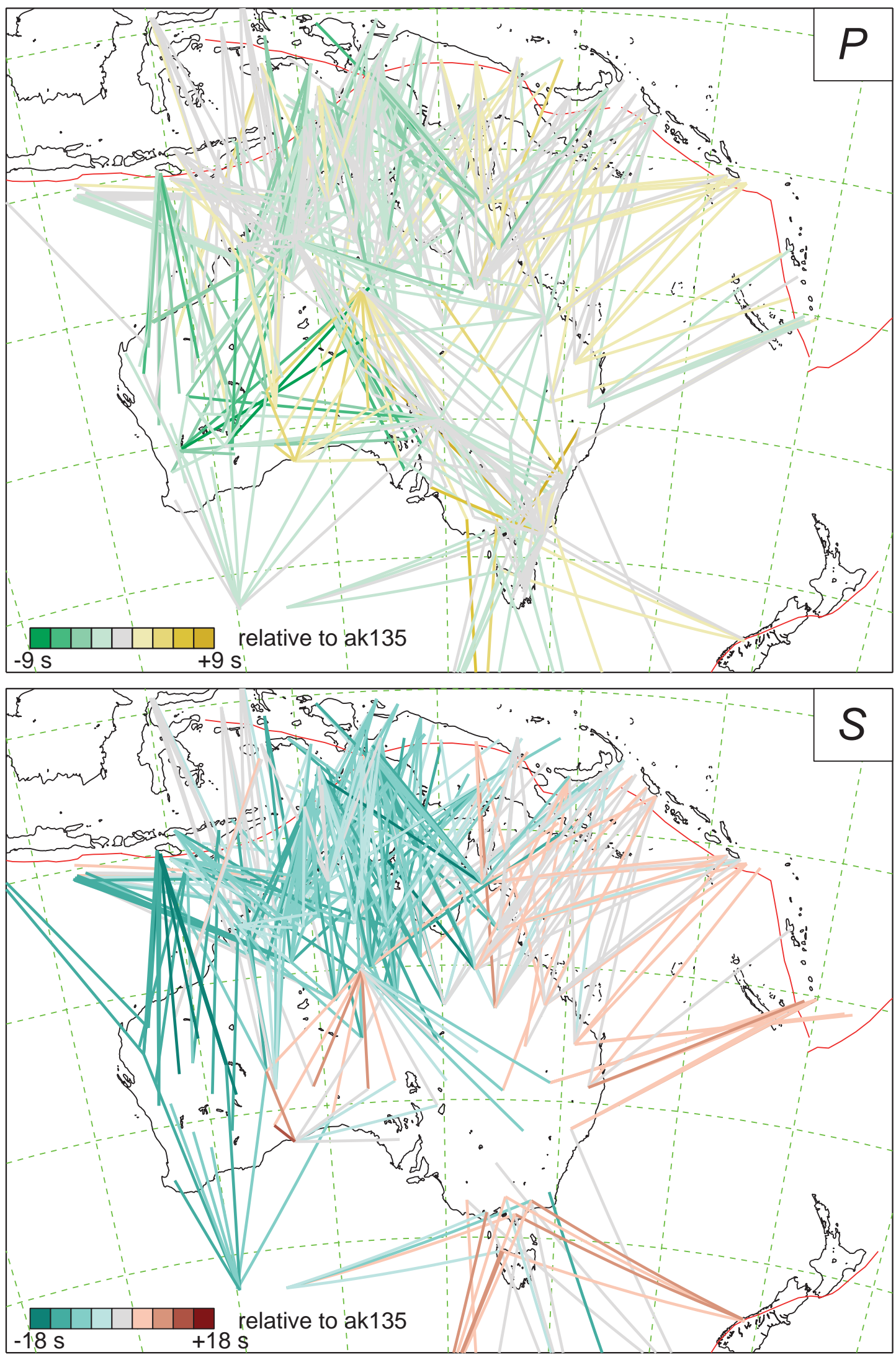

Figure 1. Event-station paths for well-located events in Australia and its environs. The $P$ and $S$ wavepaths out to $18^{\circ}$ epicentral distance mostly traverse the lithosphere. The paths are color coded by their travel-time residuals for the ak135 model. They demonstrate the strong lateral variation in seismic wavespeeds across Australia. 
reflection profiling provides detailed Moho information in parts of the continent where there is only modest control on seismic velocities. The inconsistency sometimes manifests itself by mantle-like velocities occurring just above the Moho. Such a thin high-velocity layer at the bottom of the crust, which is purely an artifact caused by the small discrepancy between Moho depth and velocity models, will lead to travel-time predictions for crustal phases that are much too short. The velocities above the Moho are therefore constrained to be less than $7.4 \mathrm{~km} / \mathrm{s}$ for $P$ waves and $4.3 \mathrm{~km} / \mathrm{s}$ for $S$ waves.

The Moho depth data in the AuSREM model do not cover the entire geographical grid employed in this paper (especially the ocean in the corners). The regions of missing data were filled in using extrapolation, so travel times for these regions are unphysical. There is no influence on any of the location results in this paper, because no relevant paths cross such regions. The 3D AuSREM model only extends to a depth of $300 \mathrm{~km}$. For this study, the 3D model has been extended below $300 \mathrm{~km}$ depth with the $1 \mathrm{D}$ model ak135 (Kennett et al., 1995).

\section{Travel-Time Computation Using the Fast Marching Method}

Three-dimensional grids of seismic travel times were precomputed for all Australian stations used in this study using the multistage fast marching method (MSFMM; de Kool et al., 2006). This method computes the first arrival time of a seismic wavefront on a set of grid nodes using a causalitybased Eikonal solver (the Fast Marching Method [FMM]) originally proposed by Sethian (1996). It is very well suited to precomputing grids of travel times to be used for location, because the grid is inherent to the method, as opposed to 3D ray-based methods that would require a ray problem to be solved for every grid point.

The implementation of MSFMM was developed for use in 3D spherical coordinates, so that the Earth's sphericity is taken into account directly and no adjustments to seismic wavespeeds are required. A special feature of the MSFMM approach is that it not only computes first arrivals but also many types of later arrivals, such as those associated with the major velocity discontinuities in the Earth. The calculation for the full suite of arrivals is achieved by dividing the computational domain into several regions (separated by the discontinuities), performing the FMM computation for each region separately, and then restarting the wavefront propagation into adjacent regions from arrival times stored at the interface between the regions. An explicit representation of the velocity discontinuities also leads to more accurate travel times as compared to a continuous representation on the same computational grid.

For this study, a computational grid resolution of $0.125^{\circ}$ in latitude and longitude, and $5 \mathrm{~km}$ in depth was used. The grid size (latitude $\times$ longitude $\times$ depth) is $269 \times 345 \times 201$ nodes, giving about 19 million nodes in total. The compu- tation of the travel-time grids for four phases (crustal $P, P n$, and the two $P$ phases turning below the 410 and $660 \mathrm{~km}$ velocity discontinuities), takes about 20 min per station on a single-processor computer. Travel-time grids are computed for both $P$ and $S$ phases. Because deep earthquakes do not occur under the Australian continent, only the top $100 \mathrm{~km}$ of the computed travel-time grids is stored for use in the location algorithm.

Evaluation of the accuracy of the MSFMM (de Kool et al., 2006) has shown that at the grid resolution used here, the numerical method can introduce travel-time errors of up to $0.3 \mathrm{~s}$ for $P$ phases, although on average these errors are much lower. These travel-time errors are considerably smaller than those introduced by uncertainty in the $3 \mathrm{D}$ velocity model.

Figure 2 shows the travel-time differences between AuSREM and the ak135 reference model for the first arriving $P$ and $S$ phases for the three stations NWAO, WRA, and CTA, spaced across the continent. The sources are set at $5 \mathrm{~km}$ depth. As expected from the observations presented in Figure 1, very large deviations in the times of passage for $P$ and $S$ are found from the ak135 predictions, up to $5 \mathrm{~s}$ for $P$ and 22 s for $S$. The calculated travel-time patterns clearly show the presence of the very fast region under western Australia in AuSREM and the rather slow region under northeast Australia. The reality of these large deviations from the ak135 model is borne out by observations, as seen in Figure 1.

The largest deviations from the ak135 reference model occur for source-receiver distances in the range between $15^{\circ}$ and $23^{\circ}$. Fifteen degrees is typically the distance at which $P n$ ray paths start to dive more deeply into the mantle rather than staying just below the Moho. Beyond $23^{\circ}$, the ray paths start to dive much more steeply through the upper mantle where most inhomogeneity occurs and interact strongly with the mantle transition zone.

\section{Location Optimization Procedure}

Once travel-time grids for each station and for all relevant phases have been precomputed, predicted travel times from an arbitrary location to the set of stations can be computed rapidly by simple trilinear interpolation in these grids. The location procedure employed here is the neighborhood algorithm (Sambridge, 1999; Sambridge and Kennett, 2001), adapted for use with the 3D AuSREM model. It is a fully nonlinear procedure with no calculation of derivatives. The method is based on an intelligent, self-focusing grid search and has been shown to be very stable and robust, although not as fast as derivative-based methods from a good starting point. An additional advantage of the method is that the trial models generated during the search can provide a robust estimate of the region of parameter space that yields acceptable solutions, and thereby the uncertainty in the result.

The essence of our nonlinear approach to event location is based on the minimization of the difference between a set of observed arrival times and those predicted from an Earth 

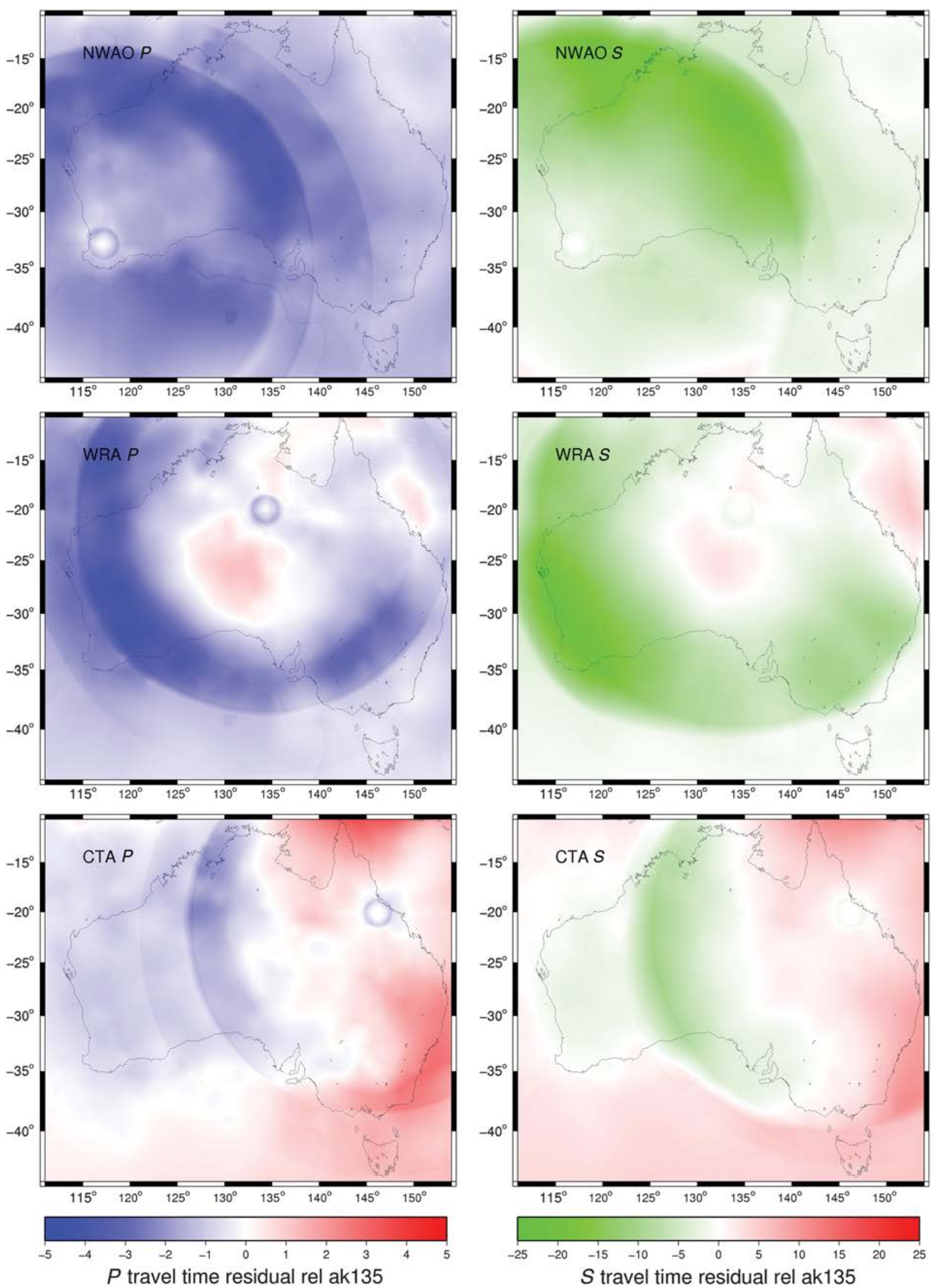

Figure 2. Travel-time residuals for first $P$ and $S$ arrivals through the Australian Seismological Reference Model (AuSREM) relative to the ak135 model. Results are shown for three stations of the Australian National Seismograph Network situated in different geologic settings. The sources are situated at $5 \mathrm{~km}$ depth. Negative residuals mean the arrival is early, positive residuals it is late. 
model. Following Billings et al. (1994), the objective function is defined as the sum of the normalized arrival-time residuals to the power 1.25 (an L1.25 norm), to reduce the effects of a small number of larger outliers as compared to the more standard L2 norm. The assumed travel-time uncertainty used to normalize arrival-time residuals in the objective function was $1.0 \mathrm{~s}$ for $P$ phases and $4.0 \mathrm{~s}$ for $S$ phases. This objective function allows for the possibility that limitations in the construction of the 3D model could lead to large residuals for some paths.

Because of the stability and robustness of the neighborhood algorithm, there is no need to start the minimization procedure from an initial location estimate obtained by independent means. The only constraint used is that the location lies within the precomputed travel-time grids. The location estimates rapidly converge to a tight patch that not only indicate the hypocenter but also provides an assessment of the constraints provided by the available data.

\section{Results}

\section{Travel-Time Residuals}

As a first test of the 3D location algorithm on the continental scale, a sample of earthquakes on the Australian continent was selected, with a preference for higher magnitude and more recent events. Events with magnitude less than 4.0 were only selected when required to increase the geographical coverage of events. Larger events were preferred as they could be observed over a range of distances, not only on local stations. The limitation to more recent earthquakes was driven by large improvements to the Australian National Seismic Network (ANSN) in terms of number and quality of stations that occurred over the last few years. Some earthquakes that occurred very close to larger events were omitted because they would not provide independent information and would bias the results to certain regions. A total of 29 events were selected. Because of the small number of events (a result of Australia's relatively low seismicity), a sample of six GT events, discussed in more detail in the Ground-Truth Events section, was added. Summary information of the events is listed in Table 1, and their locations are shown in Figure 3.

The events were located with both the ak135 and AuSREM velocity models. In both cases, we used the fully nonlinear neighborhood algorithm that provides a selffocusing grid search in 4D (latitude, longitude, depth, and origin time), with predicted travel times derived from precomputed 3D travel-time grids in each case. Because no deep earthquakes are known to occur under the Australian continent, the source depth was only allowed to range from 0 to $20 \mathrm{~km}$. The low density of the station network means there are no stations in very close proximity to the events. Because of the well-known trade-off between depth and origin time when no local station data is available, the depth of the location estimates is only poorly constrained by the observations.
The largest differences in the location estimates between the two models occur for the events located offshore in the very northern part of the region. These differences can be partly attributed to poor location geometry as the events fall outside the ANSN and also partly to limited constraints on the AuSREM model in these areas. Apart from these northern events, location differences are typically of the order of $10 \mathrm{~km}$. There is a trend for location differences to be smaller when fewer phases are available for location. This counterintuitive result is caused by the fact that fewer stations imply that the event has only been observed on nearby stations. As can be seen from the examples in Figure 2, the difference between the AuSREM and ak135 travel times is less for close distances.

In Figure 4, we display the pattern of arrival-time residuals for both $P$ and $S$ arrivals as a function of distance for the full set of event. The residuals are relative to the predictions made for the locations for the ak135 and AuSREM models from the same location procedure. Comparing the ak135 and AuSREM residuals, two main features can be noted. First, the scatter in the residuals for the AuSREM locations is significantly smaller with the reduction being much more pronounced for $S$ phases. Second, there is a clear systematic offset in the ak135 residuals, which is a function of distance and most likely associated with an origin time shift needed to minimize the measure of misfit for the location. Using the AuSREM model reduces this systematic offset, again especially for $S$ phases, but does not remove it completely. These results indicate that the AuSREM travel times are a much better representation of the true travel times for the Australian continent than the ak135 model. It is somewhat surprising that at distances beyond $23^{\circ}$, the residuals are systematically positive by a few seconds, as travel times usually become more accurate at distances beyond the triplication range because of the shorter path through the more inhomogeneous upper mantle. A possible explanation for this observation is discussed in the Ground-Truth Events section.

\section{Ground-Truth Events}

GT events (with accurately known locations) are rare in Australia because of the relatively low network density. GT locations (as based on the Bondár et al., 2004, classification) for the six events used here were derived with different techniques. Four events occurred inside dense local seismic monitoring networks. One GT location was derived from ground deformation observed with Interferometric Synthetic Aperture Radar (InSAR) and one larger earthquake ruptured to the surface (Table 2).

Figure 5 compares the mislocations of the GT events using the two velocity models. We show both the horizontal mislocations (Fig. 5a) and the deviations in depth from the GT estimates (Fig. 5b). Unfortunately, the number of events is too small to reliably identify any systematics in these results. It would appear that if the ak135 location is already good, there is not much improvement from using AuSREM. 
Table 1

The Sample of Earthquakes Used in This Study

\begin{tabular}{|c|c|c|c|c|c|c|}
\hline Date (yyyy/mm/dd) & Time (hh:mm:ss.ss) & Latitude $\left({ }^{\circ}\right)$ & Longitude $\left({ }^{\circ}\right)$ & $\Delta(\mathrm{km})^{*}$ & $N(P)^{\dagger}$ & $N(S)^{\dagger}$ \\
\hline $2006 / 05 / 13$ & $01: 04: 49.53$ & -27.86 & 135.7 & 24.96 & 12 & 10 \\
\hline $2007 / 11 / 19$ & 20:01:06.24 & -12.86 & 143.9 & 35.44 & 13 & 5 \\
\hline $2008 / 02 / 13$ & $21: 57: 28.84$ & -27.44 & 146.47 & 11.76 & 14 & 9 \\
\hline $2009 / 01 / 26$ & $00: 29: 18.99$ & -32.69 & 139.05 & 6.36 & 24 & 14 \\
\hline $2009 / 03 / 05$ & $12: 53: 50.91$ & -30.19 & 117.84 & 11.79 & 16 & 12 \\
\hline $2009 / 03 / 06$ & 01:35:59.38 & -16.34 & 121.31 & 36.58 & 20 & 16 \\
\hline $2009 / 04 / 08$ & 05:31:54.69 & -34.13 & 116.97 & 10.78 & 15 & 12 \\
\hline $2010 / 06 / 05$ & 21:46:59.17 & -33.48 & 136.69 & 11.96 & 30 & 19 \\
\hline $2010 / 07 / 30$ & $21: 29: 23.21$ & -32.23 & 138.14 & 7.51 & 26 & 14 \\
\hline $2010 / 10 / 27$ & $14: 18: 03.78$ & -29.49 & 143.75 & 8.03 & 31 & 15 \\
\hline $2011 / 02 / 21$ & 21:59:35.77 & -21.15 & 135.27 & 11.82 & 27 & 26 \\
\hline $2011 / 04 / 16$ & 05:31:18.35 & -20.16 & 147.69 & 12.98 & 29 & 3 \\
\hline $2011 / 05 / 20$ & 20:03:27.35 & -23.44 & 119.36 & 7.84 & 24 & 13 \\
\hline $2011 / 07 / 05$ & $01: 32: 12.53$ & -38.4 & 145.84 & 3.41 & 15 & 7 \\
\hline $2011 / 07 / 14$ & 05:51:36.09 & -20.92 & 120.38 & 16.06 & 25 & 14 \\
\hline $2011 / 10 / 15$ & $10: 38: 14.14$ & -32.31 & 138.82 & 9.55 & 23 & 15 \\
\hline $2011 / 12 / 05$ & $19: 10: 00.00$ & -21.5 & 114.42 & 65.17 & 22 & 16 \\
\hline $2012 / 03 / 16$ & $11: 24: 37.65$ & -26.09 & 132.08 & 17.69 & 26 & 14 \\
\hline $2012 / 11 / 05$ & $19: 31: 49.63$ & -11.78 & 129.1 & 17.34 & 26 & 20 \\
\hline $2012 / 11 / 28$ & $22: 35: 20.35$ & -27.77 & 125.93 & 3.96 & 26 & 14 \\
\hline $2013 / 06 / 09$ & $14: 22: 13.91$ & -25.92 & 132.01 & 10.81 & 33 & 30 \\
\hline $2013 / 07 / 18$ & $11: 39: 11.58$ & -19.86 & 133.88 & 9.84 & 26 & 14 \\
\hline $2013 / 08 / 21$ & $11: 02: 56.51$ & -33.46 & 117.69 & 11 & 13 & 13 \\
\hline $2013 / 08 / 24$ & $20: 27: 49.38$ & -34.31 & 148.38 & 2.5 & 15 & 8 \\
\hline $2013 / 09 / 13$ & $11: 58: 53.47$ & -20.99 & 121.02 & 16.33 & 24 & 23 \\
\hline $2013 / 09 / 30$ & $21: 14: 02.69$ & -31.84 & 121.57 & 5.66 & 20 & 6 \\
\hline $2013 / 11 / 18$ & $17: 43: 54.68$ & -16.68 & 128.72 & 3.77 & 12 & 11 \\
\hline $2013 / 12 / 12$ & $17: 24: 56.23$ & -25.59 & 139.81 & 3.24 & 16 & 13 \\
\hline $2013 / 12 / 16$ & $18: 38: 41.81$ & -21.81 & 125.28 & 16.05 & 15 & 15 \\
\hline $2007 / 10 / 09$ & $23: 58: 39.89$ & -33.94 & 117.57 & 1.8 & 11 & 9 \\
\hline $2010 / 04 / 20$ & $00: 17: 08.28$ & -30.68 & 121.47 & 3.4 & 27 & 19 \\
\hline $2012 / 03 / 23$ & 09:25:15.94 & -26.08 & 132.1 & 12.4 & 21 & 21 \\
\hline $2012 / 06 / 19$ & $10: 53: 30.40$ & -38.2 & 146.23 & 5.1 & 36 & 15 \\
\hline $2012 / 11 / 29$ & $19: 21: 22.10$ & -27.76 & 140.75 & 6.9 & 26 & 12 \\
\hline $2013 / 10 / 30$ & $22: 25: 36.66$ & -27.77 & 120.74 & 7.4 & 26 & 22 \\
\hline
\end{tabular}

$* \Delta$ is the horizontal distance between ak135 and AuSREM epicenters in kilometers. Event depth is not considered in this table because it is poorly constrained.

${ }^{\dagger} N(P)$ and $N(S)$ are the number of $P$ and $S$ phases used in the location process.

The two events with the largest AuSREM mislocations are in western Australia. The location offset is in the same direction for both models; it is only reduced in size for AuSREM. This seems to indicate that the amplitude of the velocity deviations in AuSREM relative to ak135 is still not large enough, most likely because the constraints on the structure of the far western part of the continent used in the AuSREM model are weaker than for the rest. This lack of constraints is the result of the distribution of regional earthquakes.

For one event (2013/10/30), an accurate origin time is also available. It is instructive to analyze this event in more detail. Figure 6 shows the travel-time residuals for this event, for the two velocity models, and with increasing constraints on the location. The open circles represent residuals for an unconstrained location, derived in the same way as in Figure 4. The same systematic offsets observed in Figure 4 are also present for this individual event. The plus signs represent the residuals when the event is fixed at the true epicenter but with the origin time left free. The residuals increase in size, especially for the shorter distances. This behavior is expected, because the imposition of the epicenter constraint decreases the opportunities for the location algorithm to reduce the misfit measure by only allowing adjustments in origin time, and so residuals tend to increase. Finally, the filled circles represent the true-time residuals for the correct origin time. A strong systematic effect is observed: virtually all the true residuals are negative, implying all model velocities are systematically too slow. In the case of the ak 135 location, there is a $4.5 \mathrm{~s}$ difference between the fitted origin time and the true origin time. For the AuSREM location, the difference is still $2.2 \mathrm{~s}$. Perhaps significantly, although caution is required because this is only a single event, the $P$ residuals on the two stations beyond $22^{\circ}$ are small for the AuSREM model, as would have been expected based on general experience. This observation suggests that the significantly positive residuals observed at longer distances in Figure 4 could be caused by a systematic error in the origin times 


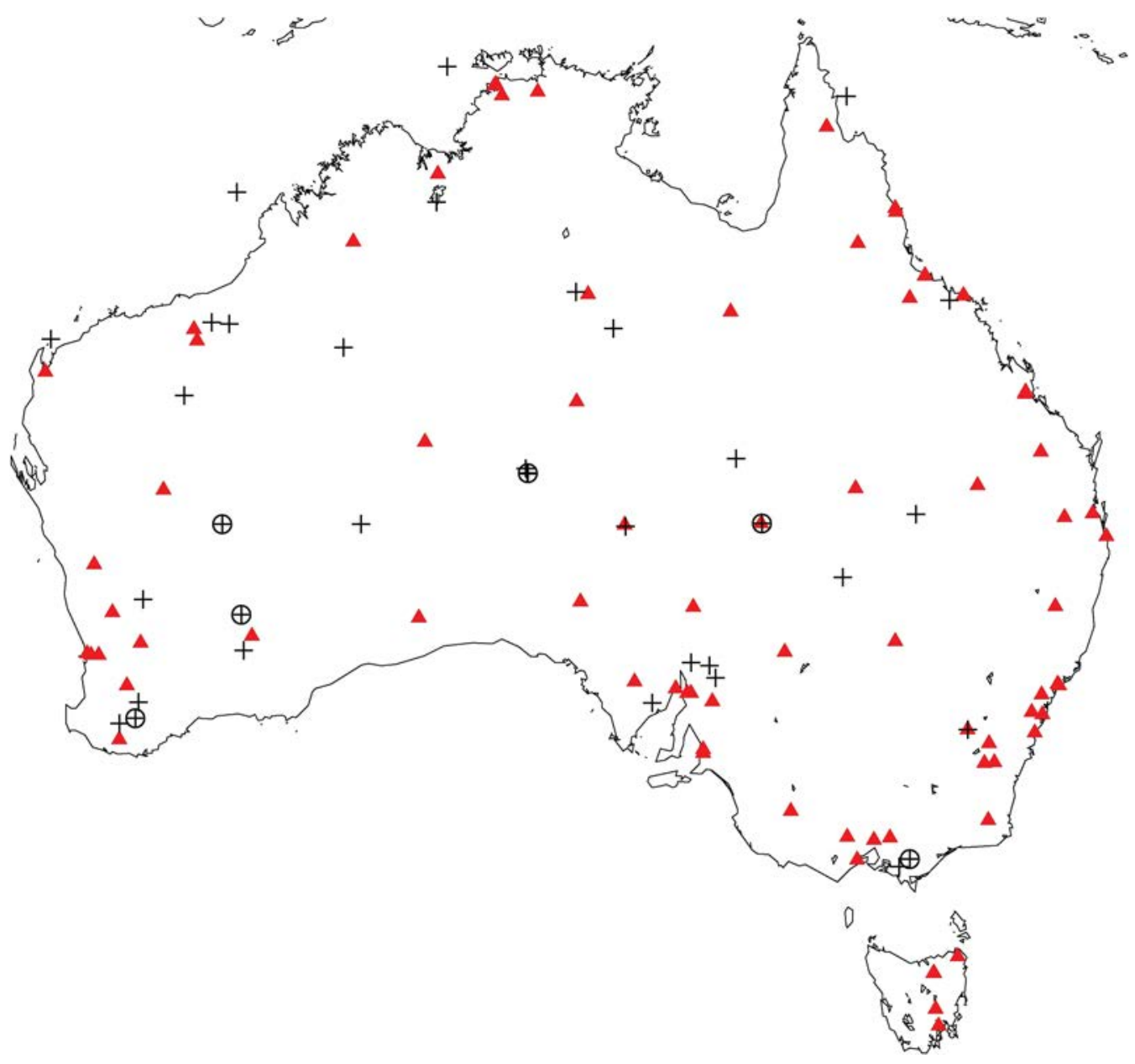

Figure 3. The locations of the earthquakes used in this study are indicated by crosses and ground-truth (GT) events are indicated by a circle. Red triangles indicate the location of the stations for which 3D travel-time grids were computed. Many stations were operational only for the more recent earthquakes in the sample. Several stations are located in urban areas and equipped with low-sensitivity instruments only.

of all events, which in turn is the result of the AuSREM velocity model not being fast enough on average.

\section{Discussion}

\section{Comparison with Geoscience Australia Earthquake Bulletin}

The method and results presented above show that it is now technically feasible to routinely locate earthquakes in Australia using the 3D AuSREM velocity model. To further evaluate the usefulness of this 3D location process, a comparison can be made with locations published in the Australian earthquake bulletin published by Geoscience Australia (GA). Many factors enter the evaluation of such a comparison because of the difference in methods employed. In the previous results, all stations with a clear arrival were used in the location procedure. The earthquake bulletin locations are derived in a highly analyst-driven process, in which arrivals on stations known to have large residuals for the source region are always omitted even if they are very clear. Furthermore, any arrival with a residual greater than $2 \mathrm{~s}$ is not used in the final location. This practice is not surprising in view of the large timing residuals demonstrated in this paper, but the strong dependence on analyst experience means it is hard to compare the performance of the location methods. The velocity models used in the GA bulletin locations are localized 1D models. The location method for the bulletin is the one provided with ANTELOPE software and relies on an initial location guess from the association grid followed by linearized minimization.

Figure 7 shows a comparison of the location offsets between AuSREM and ak135 locations and AuSREM and GA bulletin locations for the events in Table 1. In the majority of cases, the GA location, based on a much smaller number of analyst-selected picks only, is closer to the AuSREM location than the ak135 locations derived here. This result shows that 3D travel times are accurate enough over all paths to allow every associated phase to be used for location, and experience-based exclusion of certain phases is not required. Thus, using the 3D velocity model can, to a significant 

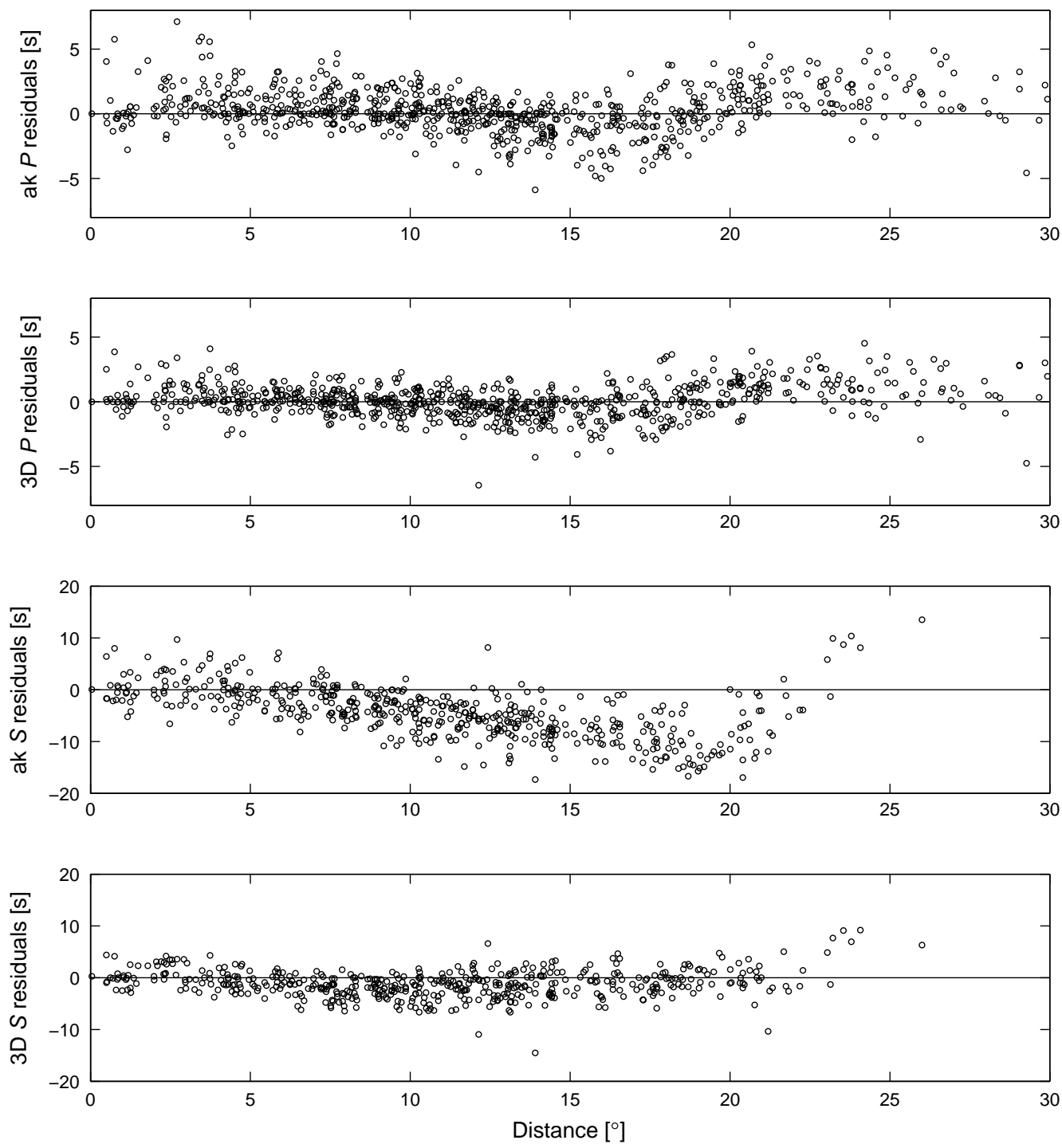

Figure 4. Travel-time residual distribution for $P$ and $S$ phases after location using the ak 135 velocity model and the AuSREM 3D velocity model. Note the difference in scale on the time residual axes for $P$ and $S$ phases.

extent, replace analyst experience and therefore lead to better locations with automated procedures.

In addition to the sample discussed in this paper, a comparison was also performed for all earthquakes in the GA bulletins from 2010 to 2012 with seven or more phase picks. This comparison was not based on independent picks but instead used exactly the same phase picks as were used for the original bulletin location. Figure 8 shows the distribution of location offsets between the AuSREM locations and the GA bulletin location. The offset is presented both in absolute terms (kilometers) and in a normalized form, in which the absolute offset is divided by the radius of the error ellipse (as derived from the AuSREM location procedure) in the direction of the location offset. Apart from a small number of outliers, the locations generally correspond well, especially when normalizing the offsets with the estimated error ellipse.
As a last test, Figure 9 compares the locations for a compact cluster of earthquakes in western Australia. The results using the AuSREM model show much tighter clustering and appear to align on well-defined fault systems. To investigate reasons for this improved performance, the same set of earthquakes was also located with the same location algorithm based on 3D travel-time grids, except with the AuSREMbased travel times replaced with ak135-based travel times. This eliminates all differences in location method except the velocity model. The result was that the cluster was as well defined as in AuSREM results but slightly shifted in position. Because the location algorithm used here gives the same tight clustering for both velocity models, it seems likely the improved performance relative to the GA bulletin is caused by the difference in location method rather than the velocity model. 

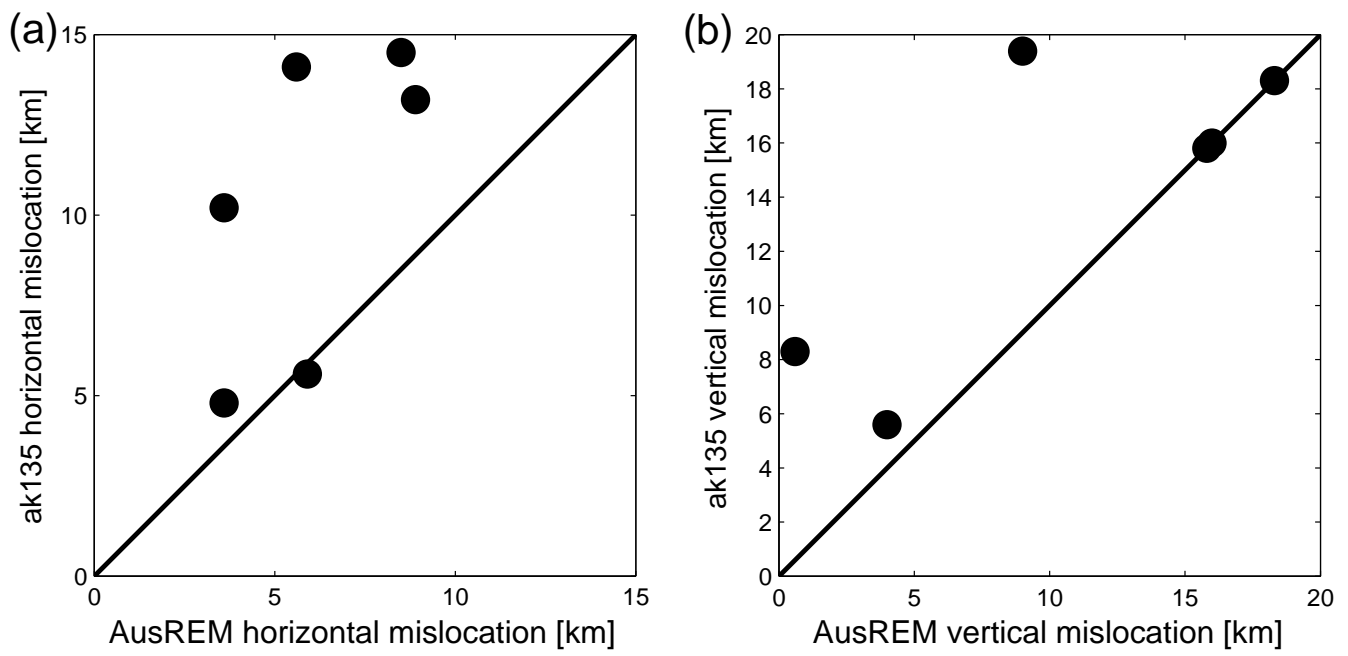

Figure 5. Comparison of (a) the horizontal and (b) the vertical mislocation of the GT events using the two velocity models. In the location procedures, the depth was only allowed to vary between 0 and $20 \mathrm{~km}$. In all cases, the error estimates indicate that there is no strongly preferred depth within the allowed $0-20 \mathrm{~km}$ range because there are no stations sufficiently close to the source.

\section{AuSREM Model Validation}

This work has demonstrated successful application of the 3D AuSREM model to locations across the continent. The location results are self-consistent, with quite small phase residuals for both $P$ and $S$ at nearly all stations. In contrast, the locations for the same events using the ak135 model display quite large residuals. The 3D locations obtained using AuSREM, in combination with the neighborhood algorithm-based search method, match well with the best results using local information, and indeed reveal features that have been obscured in earlier bulletin locations.

The primary parameter for the mantle component of AUSREM is the $S V$-wavespeed distribution, constructed by combining multiple models derived from waveform fitting and tomography using surface waves and employing bodywave constraints (Kennett et al., 2013). The $P$-wave distribution is derived from this $S V$-wavespeed model using a simple formula. For $S$-travel-time residuals derived from the AuSREM model, the strong distance bias observed in the ak135 results has almost disappeared (Fig. 4). The $P$-wave arrivals tend to become earlier with increasing distance out to $17^{\circ}$, a trend that could be corrected by a slight increase in the $P$ wavespeed for the cratonic areas.

In any form of tomography, there is a tendency for the resulting 3D wavespeed model to underestimate the actual wavespeed because of the need to regularize the solution. The absence of earthquakes to the immediate west of Australia means that the available path coverage from regional events is not as good in the west of the continent as for the rest. It appears that as a result the $S V$ wavespeeds in AuSREM are a little slower than is needed to get tight correspondence with independent evidence for event location.

On the whole, the performance of the 3D AuSREM model is very good across the whole continent. The modest deviations that remain can be taken into account when the model is revised. With the improved event locations, it is intended to expand the database of $P n$ and $S n$ observations in Australia and to revisit the wavespeed distribution in the uppermost mantle, where crustal influences can contaminate results from waveform fitting for surface waves.

Table 2

The Sample of Ground-Truth (GT) Events Used in This Study

\begin{tabular}{|c|c|c|c|c|c|c|c|c|c|}
\hline $\begin{array}{c}\text { Date } \\
\text { (yyyy/mm/dd) }\end{array}$ & $\begin{array}{c}\text { Time } \\
\text { (hh:mm:ss.ss) }\end{array}$ & $\begin{array}{l}\text { Latitude } \\
\left(^{\circ}\right)^{*}\end{array}$ & $\begin{array}{l}\text { Longitude } \\
\left({ }^{\circ}\right)^{*}\end{array}$ & $\begin{array}{l}\text { Depth } \\
(\mathrm{km})^{*}\end{array}$ & $\begin{array}{l}\Delta(1 \mathrm{D}) \\
(\mathrm{km})^{\dagger}\end{array}$ & $\begin{array}{l}\Delta(3 \mathrm{D}) \\
(\mathrm{km})^{\dagger}\end{array}$ & $N(P)^{\stackrel{े}{*}}$ & $N(S)^{\ddagger}$ & GT Method \\
\hline 2007/10/09 & $23: 58: 39.89$ & -33.95 & 117.53 & 0.6 & 4.9 & 3.7 & 11 & 9 & InSAR GT2 \\
\hline $2010 / 04 / 20$ & $00: 17: 08.28$ & -30.79 & 121.49 & 1.7 & 13.2 & 8.9 & 27 & 19 & Local network GT2 \\
\hline $2012 / 03 / 23$ & $09: 25: 15.94$ & -26.12 & 132.12 & 4 & 14.1 & 5.6 & 21 & 21 & Surface rupture GT5 \\
\hline $2012 / 06 / 19$ & $10: 53: 30.40$ & -38.25 & 146.23 & 14 & 5.6 & 5.9 & 36 & 15 & Local network GT5 \\
\hline $2012 / 11 / 29$ & $19: 21: 22.10$ & -27.80 & 140.75 & 4.2 & 10.2 & 3.6 & 26 & 12 & Local network GT1 \\
\hline $2013 / 10 / 30$ & $22: 25: 37.90$ & -27.82 & 120.70 & 0.6 & 14.5 & 8.5 & 26 & 22 & Local network GT1 \\
\hline
\end{tabular}

*Latitude, longitude, and depth refer to the GT values.

${ }^{\dagger} \Delta$ (1D) and $\Delta$ (3D) refer to the horizontal error in ak135 and AuSREM locations, respectively.

${ }^{\ddagger} N(P)$ and $N(S)$ are the number of $P$ and $S$ phases used in the location process. 

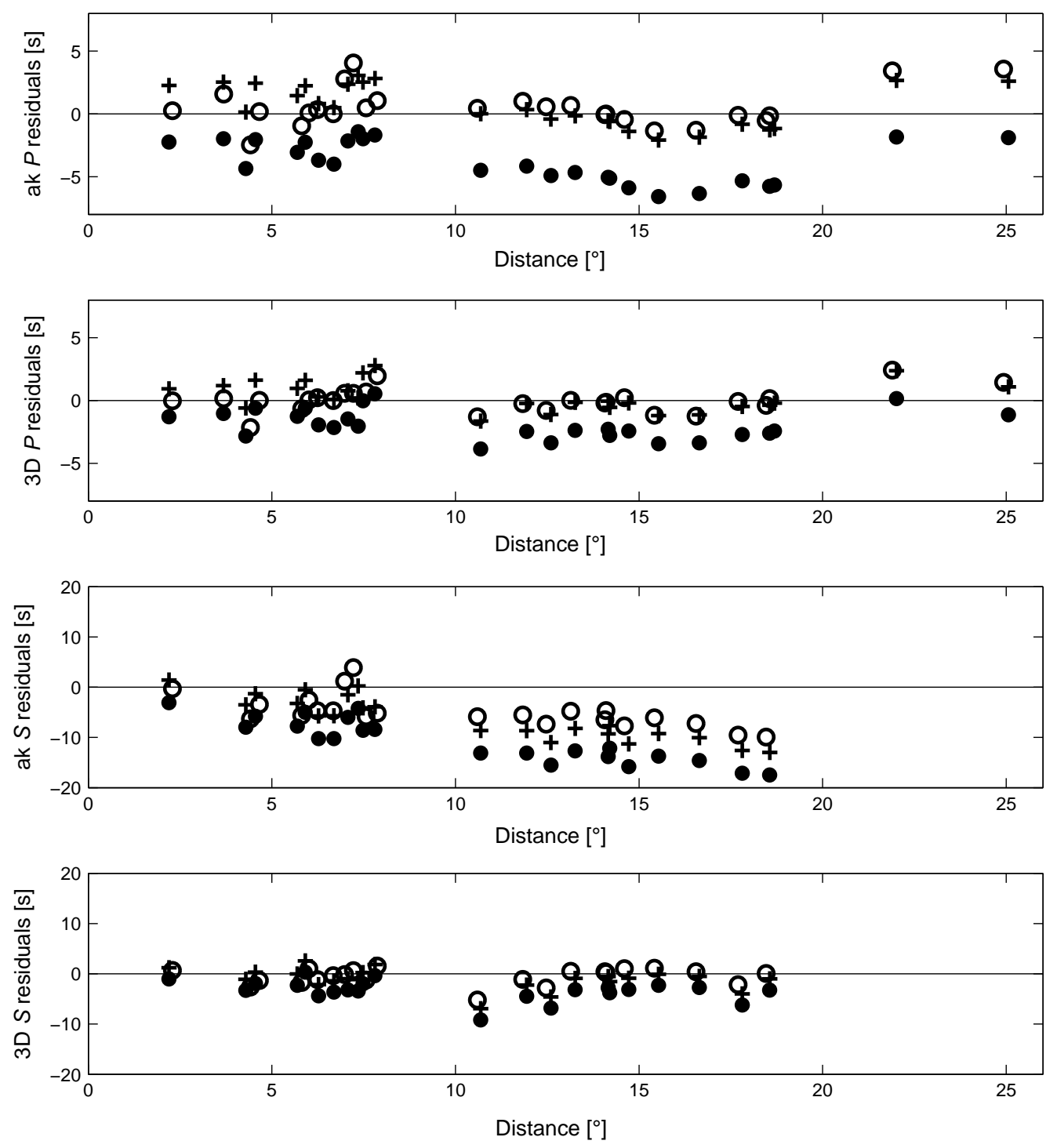

Figure 6. Travel-time residuals for the event on 30 October 2013 in western Australia. Open circles are the residuals for an unconstrained location; plus signs are the residuals when fixing the event at the GT location (i.e., optimizing only for origin time); and filled circles are the true-time residuals taking the known GT time into account.

\section{Conclusion}

It has been demonstrated that event location using a full $3 \mathrm{D}$ velocity model is both practicable and effective at the continental scale in Australia. In this work, 3D event location was achieved by exploiting the recently developed AuSREM model and incorporating this into a fully nonlinear location method. The major computational task is to develop a 3D grid of travel times for the suite of Australian stations. Once this 3D grid has been constructed using the MSFMM, travel times for both $P$ and $S$ phases can be rapidly extracted for any putative source by interpolation. The neighborhood algorithm was used to search 4D hypocenter space with a domain spanning the entire continent, using an objective function that reduces sensitivity to large residuals. This method was shown to achieve rapid convergence to a well-defined loca- tion in space and time without the need to impose any initial constraints. Only direct travel-time calculations are employed, and no derivatives are needed, which substantially reduces the computational burden for using a 3D model. In this work, attention was confined to Australian events and stations, but the neighborhood algorithm approach can also incorporate teleseismic information with allowance for 3D structure beneath Australia.

The locations achieved with the 3D AuSREM model are significantly better, and more self-consistent, than for a global model such as ak135. Indeed the accuracy achieved using a sparse network reaches, or surpasses, that for bulletin locations with strong local information. This could be of relevance for current sparse networks in operation, such as the International Monitoring System operated by the Comprehensive Nuclear-Test-Ban Treaty Organization. 

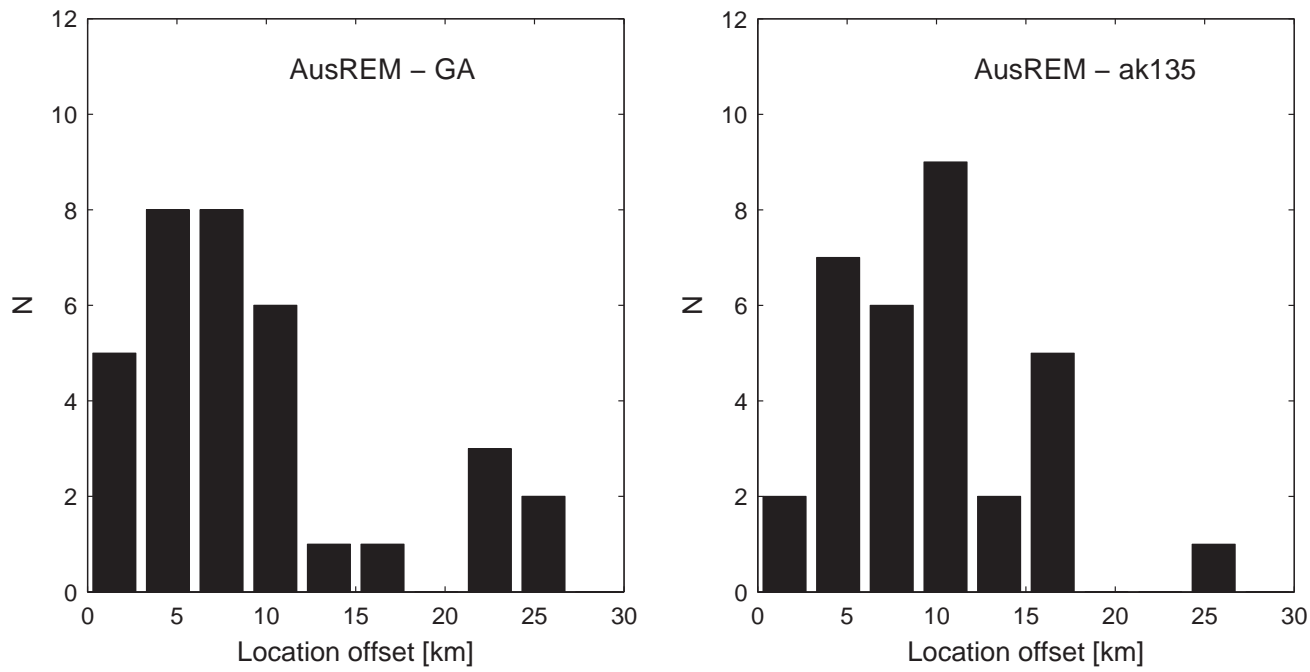

Figure 7. Location offsets between AuSREM, ak135, and GA bulletin locations for the sample in this article (Table 1).
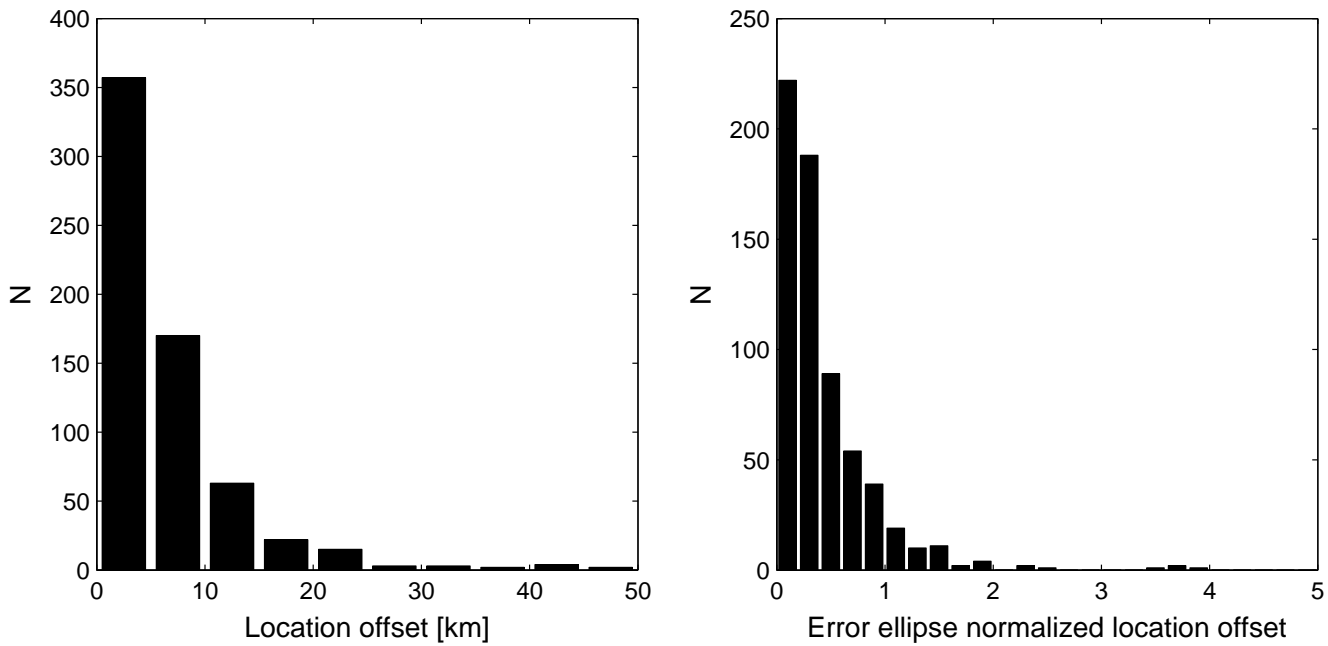

Figure 8. Location offsets between AuSREM and the GA bulletin, for GA bulletin events in the 2010-2012 period, using the same set of phase picks. Only bulletin events with seven or more defining phases are used.

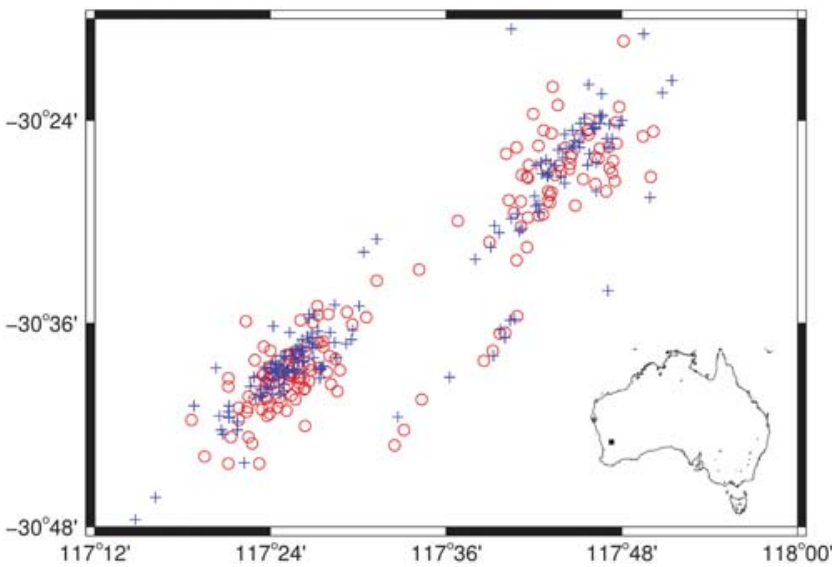

Figure 9. Map view comparison between AuSREM and GA bulletin locations for an earthquake cluster in western Australia, situated as indicated on the insert map. GA bulletin epicenters are shown with circles and AuSREM locations as crosses.
The AuSREM model has performed well in this independent test. The inclusion of further constraints from paths from continental earthquakes in Australia will allow the uppermost mantle structure to be improved and should help with correcting the slight underestimate of $S$ wavespeed in the far west of the continent.

\section{Data and Resources}

The arrival times for the events employed in the comparison between the ak135 and AuSREM model locations were picked by MdeK on waveform data from the Australian National Seismograph Network. This data is openly available through Incorporated Research Institutions for Seismology. Event information for the comparison with analyst-derived locations was taken from the Geoscience Australia earthquake bulletin (http://ga.gov.au/earthquakes, last accessed August 
2013) with the help of Spiro Spiliopoulos. The AuSREM model for both crustal and mantle wavespeeds was obtained from http://rses.anu.edu.au/seismology/AuSREM (last accessed March 2014). Some figures in this paper were produced with the Generic Mapping Tools graphics package.

\section{Acknowledgments}

The authors would like to acknowledge C. Drover (BHP Billiton Nickel West), Geodynamics Limited (R. Hogarth), and Dan Sandiford (University of Melbourne) for providing accurate ground-truth information for some of the events used. This paper is published with the permission of the CEO, Geoscience Australia.

\section{References}

Begnaud, M. L., S. Ballard, C. J. Young, J. R. Hipp, M. C. Chang, A. V. Encarnacao, C. A. Rowe, W. S. Phillips, and L. K. Steck (2011). A global 3D P-velocity model of the Earth's crust and Mantle for improved event location, Proceedings of the 2011 Monitoring Research Review, Tucson, Arizona, 13-15 September 2011, 237-248.

Billings, S., B. L. N. Kennett, and M. S. Sambridge (1994). Hypocentre location: Genetic algorithms incorporating problem specific information, Geophys. J. Int. 118, 693-706.

Bondár, I., S. C. Myers, E. R. Engdahl, and E. A. Bergman (2004). Epicenter accuracy based on seismic network criteria, Geophys. J. Int. 156, 483-496.

Chen, H., J.-M. Chiu, J. Pujol, K. Kim, K.-C. Chen, B.-S. Huang, Y.-H. Yeh, and S.-C. Chiu (2006). A simple algorithm for local earthquake location using 3D $V_{P}$ and $V_{S}$ models: Test examples in the central United States and in central eastern Taiwan, Bull. Seismol. Soc. Am. 96, 288-305.

de Kool, M., N. Rawlinson, and M. Sambridge (2006). A practical grid-based method for tracking multiple refraction and reflection phases in threedimensional heterogeneous media, Geophys. J. Int. 167, 253-270.

Husen, S., E. Kissling, N. Deichmann, S. Wiemer, D. Giardini, and M. Bae (2003). Probabilistic earthquake location in complex three-dimensional velocity models: Application to Switzerland, J. Geophys. Res. 108, doi: 10.1029/2002JB001778.

Kennett, B. L. N. (2006). On seismological reference models and the perceived nature of heterogeneity, Phys. Earth Planet. In. 159, 129-139.

Kennett, B. L. N., and E. R. Engdahl (1991). Travel times for global earthquake location and phase identification, Geophys. J. Int. 105, 429-465.

Kennett, B. L. N., and M. Salmon (2012). AuSREM: Australian seismological reference model, Aust. J. Earth Sci. 59, 1091-1103.

Kennett, B. L. N., E. R. Engdahl, and R. Buland (1995). Constraints on seismic velocities in the Earth from travel times, Geophys. J. Int. 122, 108-124.

Kennett, B. L. N., A. Fichtner, S. Fishwick, and K. Yoshizawa (2013). Australian seismological reference model (AuSREM): Mantle component, Geophys. J. Int. 192, 871-887.
Kennett, B. L. N., M. Salmon, E. Saygin, and AusMoho Working Group (2011). AusMoho: The variation in Moho depth in Australia, Geophys. J. Int. 187, 946-958.

Lomax, A., A. Zollo, P. Capuano, and J. Virieux (2001). Precise, absolute earthquake location under Somma Vesuvius volcano using a new three-dimensional velocity model, Geophys. J. Int. 146, 313-331.

Molinari, I., and A. Morelli (2011). EPcrust: A reference crustal model for the European plate, Geophys. J. Int. 185, 352-364, doi: 10.1111/ j.1365-246X.2011.04940.x.

Salmon, M., B. L. N. Kennett, and E. Saygin (2013). Australian seismological reference model (AuSREM): Crustal component, Geophys. J. Int. 192, 190-206.

Salmon, M., B. L. N. Kennett, T. Stern, and A. R. A. Aitken (2013). The Moho in Australia and New Zealand, Tectonophysics 609, 288-298.

Sambridge, M. S. (1999). Geophysical inversion with a neighbourhood algorithm I. Searching a parameter space, Geophys. J. Int. 138, 479-494.

Sambridge, M. S., and B. L. N. Kennett (2001). Seismic event location: nonlinear inversion using a neighbourhood algorithm, Pure Appl. Geophys. 158, 241-259.

Satriano, C., L. Elia, C. Martino, M. Lancieri, A. Zollo, and G. Iannaccone (2011). PRESTo, the earthquake early warning system for Southern Italy: concepts, capabilities and future perspectives, Soil Dynam. Earthq. Eng. 31, 137-153.

Schivardi, R., and A. Morelli (2011). EPmantle: A three-dimensional transversely isotropic model of the upper mantle under the European Plate, Geophys. J. Int. 185, 469-484, doi: 10.1111/j.1365-246X.2011.04953.x.

Sethian, J. A. (1996). A fast marching level set method for monotonically advancing fronts, Proc. Natl. Acad. Sci. Unit. States Am. 93, no. 4, $1591-1595$

Simmons, N. A., S. C. Myers, G. Johannesson, and E. Matzel (2012). LLNLG3Dv3: Global P wave tomography model for improved regional and teleseismic travel time prediction, J. Geophys. Res. 117, no. B10302, doi: 10.1029/2012JB009525.

Yuan, H., and B. Romanowicz (2010). Lithospheric layering in the North American craton, Nature 466, 1063-1068.

Geoscience Australia

GPO Box 378

Canberra ACT 2601, Australia

marthijn.dekool@ga.gov.au (M.d.K.)

Research School of Earth Sciences

The Australian National University

Canberra ACT 0200, Australia (B.L.N.K.)

Manuscript received 15 April 2014; Published Online 21 October 2014 